\title{
Flexible Certificate Revocation List for Efficient Authentication in loT
}

\author{
Li Duan \\ Paderborn University \\ Germany \\ liduan@mail.upb.de
}

\author{
Yong Li \\ Huawei Technologies \\ Germany \\ Yong.Li1@huawei.com
}

\author{
Lijun Liao \\ Huawei Technologies \\ Germany \\ lijun.liao@gmail.com
}

\begin{abstract}
When relying on public key infrastructure (PKI) for authentication, whether a party can be trusted primarily depends on its certificate status. Bob's certificate status can be retrieved by Alice through her interaction with Certificate Authority (CA) in the PKI. More specifically, Alice can download Certificate Revocation List (CRL) and then check whether the serial number of the Bob's certificate appears in this list. If not found, Alice knows that Bob can be trusted. Once downloaded, a CRL can be used offline for arbitrary many times till it expires, which saves the bandwidth to an extreme. However, if the number of revoked certificates becomes too large, the size of the CRL will exceed the RAM of Alice's device. This conflict between bandwidth and RAM consumption becomes even more challenging for the Internet-of-Things (IoT), since the IoT end-devices is usually constrained by both factors.

To solve this problem in PKI-based authentication in IoT, we proposed two novel lightweight CRL protocols with maximum flexibility tailored for constrained IoT end-devices. The first one is based on generalized Merkle hash tree and the second is based on Bloom filter. We also provided quantitative theorems for CRL parameter configuration, which help strike perfect balance among bandwidth, RAM usage and security in various practical IoT scenarios. Furthermore, we thoroughly evaluated the proposed CRL protocols and exhibited their outstanding efficiency in terms of RAM and bandwidth consumption. In addition, our formal treatment of the security of a CRL protocol can also be of independent interest.
\end{abstract}

\section{Author Keywords}

Authentication, Internet-of-Things, Certificate Revocation List, Public Key Infrastructure, provable security

\section{INTRODUCTION}

Mass deployment of Internet-of-Things (IoT) end devices is an irreversible trend [1]. Billions of devices online mean tens of billions of connections per day. On the other hand, data security is critical in IoT. Failing to deploy confidentiality protection or authentication mechanism in IoT system has already

\footnotetext{
Permission to make digital or hard copies of part or all of this work for personal or classroom use is granted without fee provided that copies are not made or distributed for profit or commercial advantage and that copies bear this notice and the full citation on the first page. Copyrights for third-party components of this work must be honored. For all other uses, contact the owner/author(s).

IOT '18 October 15-18, 2018, Santa Barbara, CA, USA

(C) 2018 Copyright held by the owner/author(s).

ACM ISBN 978-1-4503-6564-2/18/10.

DOI: https : //doi .org/10.1145/3277593. 3277595
}

resulted in multiple privacy-breaching incidents [20]. Therefore, unless in a fully trusted environment, the IoT device (client) and the IoT platform (server) have to apply authenticated key exchange (AKE) [2] or authenticated and confidential channel establishment (ACCE) protocols [12] for secure communication against adversaries, i.e., to use protocols through which only the intended talking partners can correctly read each message. A typical ACCE protocol, such as TLS 1.2 [11], requires the participants to authenticate each other via certificate checking, as shown in Figure 1. In general, the authentication procedure can be implemented with either symmetric cryptography or public key cryptography.

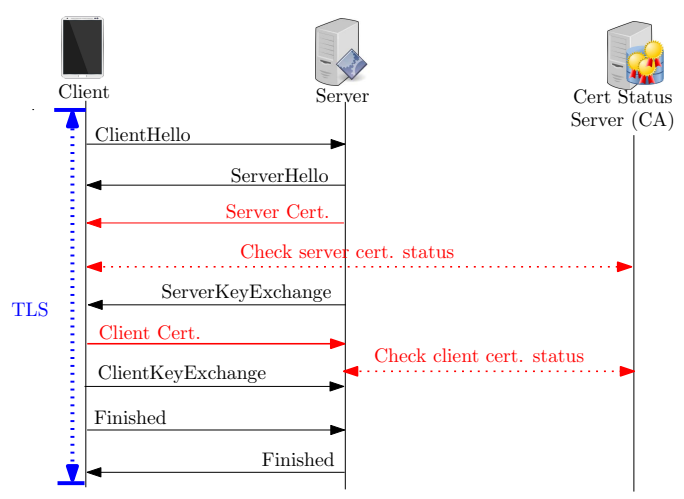

Figure 1: Certificate validation in TLS 1.2

Authentication via symmetric cryptography. Long term secret key materials are distributed securely to each communicating partners during the setup phase. This can be done offline by using physical tokens, for instance, with a smart card or eFUSE. The secret is burnt into the tokens and both the client and the server share the same secret serving as a root of trust for authentication and the transport of application data, if symmetric cryptography is used as in TLS-PSK [9]. Though easy to implement and efficient in computation and communication, managing massive number of symmetric secret keys can generate a considerable amount work for the IoT platform (for example, frequent search over one billion keys stored in the database) and might create a single point of failure in the authentication sub-system. Once the keys are leaked from the server side, every single client secret key has to be patched to keep the end-device secure.

Authentication via PKI with public key cryptography. Another way of distributing long-term secret online involves a certifica- 
tion authority (CA), which is part of a public key infrastructure (PKI) as in Figure 1. In this paper, we focus on the scenarios with authentication using PKI. This approach can greatly simplify the key management on the server side and since the server does not hold the client's secret, even if the server's secret key is leaked, the clients only need to update their storage of the server's public key.

When relying on PKI for the authentication, whether a party can be trusted primarily depends on its certificate status. The peer's certificate status can be retrieved by the user via interacting with Certificate Authority (CA) in the PKI. There are two predominant methods for doing so. The user can download Certificate Revocation List (CRL) and then check if the peer's certificate is in the CRL, or the user can directly use Online Certificate Status Protocol (OCSP) [7] and obtain a status value from the CA confirming the (in-)validity of the server's certificate. The client will terminate the connection if server certificate is found revoked.

Using OCSP. If OCSP is used, the user will first send a request to the OCSP responder for the status of the peer's certificate. The response is signed by the CA, which contains a yes-no answer $^{1}$, as well as the single serial number of the peer's certificates. Though the size of OCSP messages is ignorable compared to a long CRL, using OCSP requires the user to be online for sending and receiving OCSP messages. On the other hand, when the number of users becomes huge, the OCSP responder might be overwhelmed by the flood of simultaneous certificate status requests. Furthermore, conventional OCSP reveals to the responder or CA the connection history of the user, leading to potential privacy risk.

Advantage and challenges of CRL. If CRL is used, the client will download a list of serial numbers of invalid certificates with augmented information signed by the CA. Once the client has downloaded a CRL, he can use it without connecting to CA. However, the disadvantage of CRL is also obvious due to its size. For example, the size of the CRL published by Apple already exceeds $26 \mathrm{MB}^{2}$. Considering the constrained capacity of an IoT end devices (RAM size between $1 \mathrm{~KB}$ to 50 $\mathrm{KB}$, see Table 1), this might prevent the use of conventional CRL.

\begin{tabular}{|c|c|c|c|}
\hline & Class 0 & Class 1 & Class 2 \\
\hline Data Size (RAM) & $\ll 10 \mathrm{~KB}$ & $\approx 10 \mathrm{~KB}$ & $\approx 50 \mathrm{~KB}$ \\
\hline Code Size (ROM) & $\ll 100 \mathrm{~KB}$ & $\approx 100 \mathrm{~KB}$ & $\approx 250 \mathrm{~KB}$ \\
\hline
\end{tabular}

Table 1: RAM / ROM size of IoT devices of each class [4]

Unlike previous work that focused on high-level innovation in architecture, such as using blockchain [13] or other distributed ledgers, we enhance the efficiency of the authentication framework through a bottom-up approach. By providing lightweight

\footnotetext{
${ }^{1}$ In fact, OCSP [7] specifies three status good, revoked and unknown. The reason why an unknown status is returned is that the responder cannot determine the status of the certificate, but unknown can indicate that the certificate queried should not be trusted.

${ }^{2}$ http://developer . apple. com/certificationauthority/wwdrca. crl The number of revoked certificates was around 668,000 in June, 2018.
}

security components, i.e., lightweight CRL to PKI for IoT, an observable efficiency improvement of authentication phase can be achieved. This paper focused on the following security and efficiency challenges.

- Optimal size of downloaded content There are two extreme cases. A complete CRL might be too large for the memory of an IoT device, while too frequent retrieval of single certificate status generates bottlenecks of the CA. Slicing the complete CRL can definitely help but the question is how to determine the size of each slice such that the end-device has enough memory to hold it while the retrieval frequency is reduced and the original security is intact.

\section{- Balance between efficiency and Integrity of the CRL} There are also two extreme cases. Conventional CRL contains only one signature but the content is too large. On the other hand, If every single item (serial number) is signed by the CA, then the size of the CRL slice can be freely adjusted by the CA and optimized for download. However, the IoT device has to spend a lot of power on verifying every signature in the slice to confirm its integrity, shortening its battery life.

We will further elaborate the challenges and form several theorems regarding the parameters for optimal efficiency in the following sections.

\section{Our Contribution and the Outline of the Paper}

We designed two lightweight CRL protocols for IoT devices, which can be parametrized to optimally strike the balance between efficiency and security under various condition. The quantitative theorems were also formed for better guidance in practice. The security proof, instantiation and evaluation showed that our design is solid and extensible.

The notations in the algorithms and definitions of cryptographic primitives are introduced in Section 2. The communication and security models for our design of lightweight CRL are presented in Section 3. The concrete design and theorems for security and performance are provided in Section 4 . The evaluation of the CRL protocols is presented in Section 5. The related works are summarized in Section 6. The conclusion and the future work are presented in Section 7.

\section{NOTATION AND PRELIMINARIES}

\section{Notation}

We let $\kappa \in \mathbb{N}$ denote the security parameter and $1^{\kappa}$ the string that consists of $\kappa$ ones. Let $[n]=\{1, \ldots, n\} \subset \mathbb{N}$ be the set of integers between 1 and $n$. If $S$ is a set, $a \stackrel{\$}{\leftarrow}$ denotes the action of sampling a uniformly random element from $S$. If $\mathscr{A}()$ is an algorithm, $m \stackrel{\$}{\leftarrow} \mathscr{A}^{\mathrm{O}(\cdot)}()$ denotes that $\mathscr{A}$ (probabilistically) outputs $m$ with the help of another algorithm $\mathrm{O}(\cdot)$. Let $X \| Y$ denote the operation concatenating two binary strings $X$ and $Y$. We use $\operatorname{Pr}[A: B]$ to denote the probability that $B$ happens if action $A$ is taken. We use $\# E$ to denote the number of objects in category $E$. 


\section{Cryptographic primitives}

DEFINITION 1. (Signature scheme) A signature scheme $\mathrm{SIG}=($ SIG.Gen, SIG.Sign, SIG.Vfy) consists of three algorithms SIG.Gen,SIG.Sign and SIG.Vfy described as below.

- SIG.Gen $\left(1^{\kappa}\right) \stackrel{\$}{\rightarrow}(\mathrm{pk}, \mathrm{sk})$. The non-deterministic key generation algorithm SIG.Gen() takes the security parameter $1^{\kappa}$ as the input and outputs the public key $\mathrm{pk}$ and the private key sk.

- SIG.Sign $(\mathrm{sk}, m) \stackrel{\$}{\rightarrow} \sigma$. The (non-deterministic) message signing algorithm SIG.Sign() takes the private key sk and a message $m$ as the input and outputs the a signature $\sigma$.

- SIG.Vfy $(\mathrm{pk}, m, \sigma)=b$. The deterministic signature verification algorithm SIG. Vfy() takes the public key pk, a message $m$ a signature $\sigma$ as input and outputs a boolean value $b . b$ is TURE iff $\sigma$ is a valid signature on $\mathrm{m}$.

DEFINITION 2. (Strongly unforgeable signature) Let $\mathrm{O}_{s k}^{\mathrm{SIG}}(\cdot)$ be a signing oracle that outputs a signature $\sigma$ given message $m$ with the private key sk in accordance to SIG. We say that a signature scheme SIG is strongly existentially unforgeable against chosen message attack if there exists a negligible function $\varepsilon_{\text {seuf }}()$ such that for any algorithm $\mathscr{A}$ with access to $\mathrm{O}_{\text {sk }}^{\mathrm{SIG}}(\cdot)$ and its running time bounded by poly $(\kappa)$ it holds that

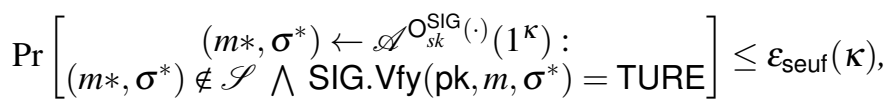

where $\mathscr{S}$ is the set of message-signature pair $\mathscr{A}$ has queried and received from $\mathrm{O}_{s k}^{\mathrm{SIG}}(\cdot)$.

DEFINITION 3. (Collision-resistant Hash Function) A hash function $H: \mathscr{M} \rightarrow \mathscr{D}$ is collision resistant if there exists a negligible function $\varepsilon_{\text {coll }}()$ such that for any algorithm $\mathscr{A}$ with running time bounded by poly $(\kappa)$, it holds that

$$
\operatorname{Pr}\left[\begin{array}{c}
\left(m_{0}, m_{1}\right) \leftarrow \mathscr{A}\left(1^{\kappa}, H\right): \\
m_{0} \neq m_{1} \wedge H\left(m_{0}\right)=H\left(m_{1}\right)
\end{array}\right] \leq \varepsilon_{\text {coll }}(\kappa),
$$

where $\mathscr{M}$ is the message space and $\mathscr{D}$ is the hash image space.

\section{COMMUNICATION AND SECURITY FRAMEWORK}

\section{Communication Settings}

We consider a general and relatively abstracted communication setting for our CRL protocols. The major parties participating in a CRL protocol include the CRL user, who is normally an IoT end device, and the CA, who is the CRL issuer. The download server is kept in Figure 2 for completeness since such a service is usually set up for off-loading the CA from storage service. As depicted in Figure 2, whenever a new CRL is issued, CA will push that CRL to the download server. The CRL user, here an IoT device, will request a CRL from the download server when his old CRL has expired or it is without sufficient information.

\section{Syntax and Security Model of CRL}

Intuitively, we would like to have a CRL in which any malicious modification can be detected by the CRL user. By malicious modification we mean the insertion and deletion of

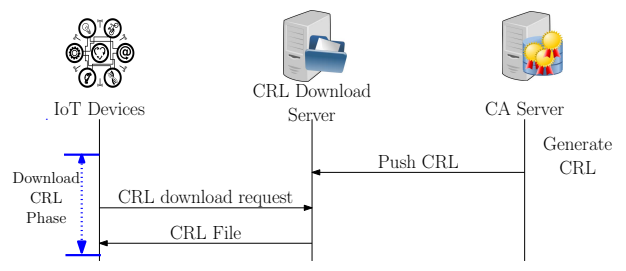

Figure 2: CRL communication sequence

certificate information, replacement of the issuer information or other auxiliary information done by the attacker. For the soundness of our CRL design, we have to formally define what to protect and how to evaluate the effect of protection. Therefore, instead of viewing CRL simply as pieces of static data, we define the CRL protocol with the following syntax.

DEFINITION 4. (Abstract CRL, the list) An abstract Certificate Revocation List $\mathrm{crl}_{(j, t)}$ issued by issuer $j$ at time point $t$, is a tuple of the following format.

$\operatorname{crl}_{(j, t)}:=\left(\right.$ certSet $_{t}=\left\{\operatorname{clD}_{i}, \text { aux }_{i}\right\}_{i \in[\mathrm{m}]}$, , issuerlnfo $_{j}$, secTag $)$, where $\mathrm{cID}_{i}$ is the unique identifier of the $i$-th revoked certificate, $\mathrm{m}$ the total number of revoked certificates at time $t$, aux the auxiliary information about the revoked certificate, issuerlnfo $_{j}$ the information of the issuer $j$ and secTag the tag containing security information associated with $\mathrm{crl}_{t}$.

DEFINITION 5. (Abstract CRL, the protocol) An abstract CRL protocol consists of the following algorithms.

- CRL.Init $\left(1^{\kappa}, j\right) \stackrel{\$}{\rightarrow}\left(\left(\mathrm{ik}_{j}, \mathrm{vk}_{j}\right), \mathrm{st}_{j}^{0}\right)$ The non-deterministic initialization algorithm CRL.Init() takes the security parameter $1^{\kappa}$ and the issuer identity $j$ as the input and outputs a pair of issuing and verification keys (ik, vk) and the initial internal state $\mathrm{st}_{j}^{0}$ for CRL issuer $j$.

- CRL.Issue $\left(\mathrm{ik}_{j}, \mathrm{st}_{j}^{t-1}\right.$, certSet $\left._{t}\right)=\left(\mathrm{st}_{j}^{t}, \mathrm{crl}_{(j, t)}\right)$ The deterministic algorithm $\mathrm{CRL}$.Issue() takes the secret key $\mathrm{ik}_{j}$, the internal state $\mathrm{st}_{j}^{t-1}$ of $j$ at $(t-1)$ and a set of certificate information certSet $t_{t}$ at time $t$ as the input and outputs the new internal state $\mathrm{st}_{j}^{t}$ and the $C R L \mathrm{crl}_{(j, t)}$.

- CRL.Verify $\left(\mathrm{vk}_{j}, \mathrm{crl}\right)=b$ The deterministic tag verification algorithm CRL.Verify() takes the verification key $\mathrm{vk}_{j}, a$ $C R L \mathrm{crl}$ as input and outputs a boolean value $b . b$ is TURE iff $\mathrm{crl}$ is a valid CRL issued by issuer $j$.

The ability of the adversary. The adversary $\mathscr{A}$ attacking the CRL protocol can control multiple CRL users but NONE of the CRL issuers (CA). Furthermore, $\mathscr{A}$ can eavesdrop, drop or reorder any CRL messages transferred over the network, but cannot modify the content of the messages sent or received by honest CRL users.

Formally, a CRL protocol should satisfy the following security definition under $\mathscr{A}$ 's attack.

DEFINITION 6. (Integrity of a CRL protocol) Let $\mathrm{O}_{\mathrm{crl}}(\cdot, \cdot, \cdot)$ be an oracle that outputs a $C R L \mathrm{crl}_{(j, t, \mathrm{certSet})}$ given 
a issuer identifier $j$, time point $t$ and a set of revoked certificates certSet. We say that a CRL has integrity if there exists a negligible function $\varepsilon_{\mathrm{int}}()$ such that for any algorithm $\mathscr{A}$ with its running time bounded by poly $(\kappa)$ it holds that

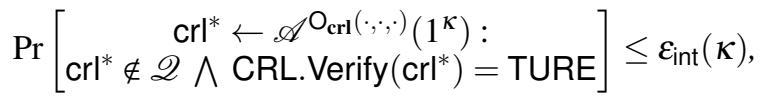

where $\mathscr{Q}$ is the set of responses $\mathscr{A}$ has received from $\mathrm{O}_{\mathrm{crl}}(\cdot, \cdot)$.

Rationale of the security definition. The integrity requires that even if an adversary has seen a lot of CRL data, he cannot modify an existing CRL or forge a new CRL which can pass the verification. The oracle $\mathrm{O}_{\mathrm{crl}}(\cdot, \cdot, \cdot)$ generalizes all the feasible way that an adversary can use to obtain any CRL with free choices of the issuer, the time and the set of certificates to be revoked.

\section{LIGHTWEIGHT CRL PROTOCOLS}

From this section on, we use the term CRL for both the list of revoked certificates and the protocol that produces the list, as it greatly simplifies the presentation. The exact meaning of this term can be inferred directly from the context.

We will present two flexible CRL protocols in the following sub-sections. The first one is called Hierarchical CRL (HCRL), in which we use a generalized Merkle hash tree [16] to reduce the RAM needed for CRL usage on a end devices at price of moderate communication complexity. The other protocol BfCRL is based on Bloom filter, which keeps the RAM usage almost constant and provides efficient search with complexity negligibly close to $\mathscr{O}(1)$.

\section{Hierarchical CRL with High Flexibility}

The main idea of HCRL is to organize the information of revoked certificate into a hierarchical data structure, a generalized Merkle hash tree [16]. To generate a hierarchical CRL, the CA first sorts the serial numbers of revoked certificates, then it divides all the serial numbers into groups. As long as the group in the current level is too large for the CRL user, CA will further divide the group to form a new level. Finally, the CA will calculate the hash values from bottom up to form a hash list and use digital signature to protect the top-level hashes. Let us see one concrete examples to illustrate our idea before the formal description.

We define the top-level as level 0 and the bottom level as level B. As shown in Figure 3, at level $B=1$, each leaf block $\mathrm{sCRL}_{\alpha}^{\mathrm{B}}$ contains a list of serial numbers $S N$ and when the certificate with this $S N$ was revoked. The minimal $S N_{\alpha, 1}^{\mathrm{B}}$, maximal $S N_{\alpha, \max }^{\mathrm{B}}$ and the hash value $H\left(\mathrm{SCRL}_{\alpha}^{\mathrm{B}}\right)$ of each complete block $\mathrm{SCRL}{ }_{\alpha}^{\mathrm{B}}$ are collected at the level above, here directly at level 0 . We call the level 0 list the master CRL. After collecting all the hashes of the blocks, the CA will hash this set of information to get a hash value $H(\mathrm{mData})$ and generate a signature $\sigma$ on $H$ (mData).

The user will use HCRL in a top-down way as shown in Figure 4. First he checks if his current CRL is expired. If so, the user will download the new master CRL. Next, he computes the hash value $h$ (mData) over the collection of

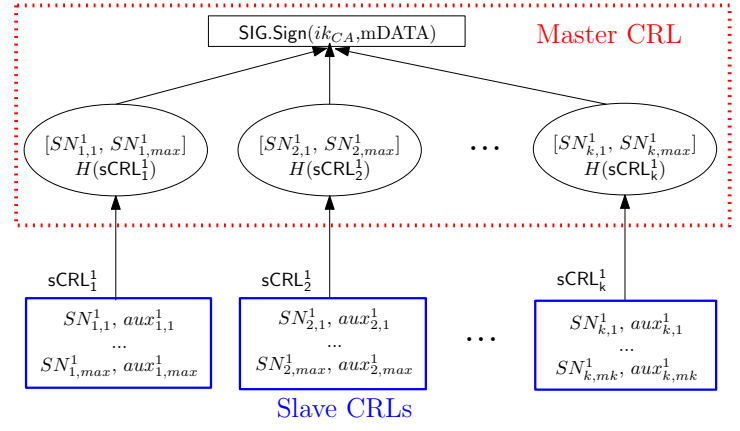

Figure 3: $H C R L$ tree structure

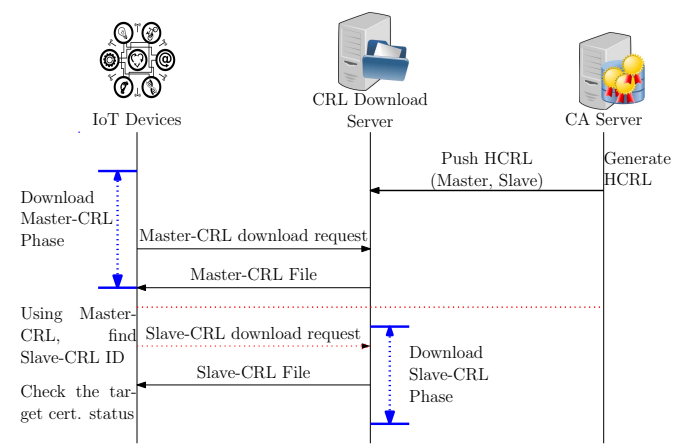

Figure 4: HCRL communication sequence

$\left\{S N_{\alpha, 1}^{\mathrm{B}}, S N_{\alpha, \text { max }}^{\mathrm{B}}, H\left(\mathrm{sCRL}_{\alpha}^{\mathrm{B}}\right)\right\}$ and verifies if $\sigma$ is a valid signature on $H$ (mData). If the verification confirms the validity of the master CRL, the user keeps it in his RAM for later use. Once he wants to check a certificate $c^{*}$, he first checks if the serial number $S N^{*}$ of the $c^{*}$ falls in an interval $\left(S N_{\alpha, 1}^{\mathrm{B}}, S N_{\alpha, \max }^{\mathrm{B}}\right)$ for any $\alpha$. If not, he knows that $c *$ is not revoked and the party corresponding to $c^{*}$ can be trusted. Otherwise he will download the block $S C R L_{\alpha}^{\mathrm{B}}$ to directly search if $S N^{*}$ is in it. No matter found or not, the final step is to verify the integrity of the CRL. Here the user hash to check whether the hash value of the block is equal to $H\left(\mathrm{sCRL}_{\alpha}^{\mathrm{B}}\right)$ stored in $\mathrm{mCRL}$. This check guards the user against CRL forgery and block replacement attacks.

Now we start formal description of each component in HCRL. At the bottom-level B, each CRL block has the form

$$
\mathrm{sCRL}^{\mathrm{B}}:=\left\{\left(S N_{s}, \operatorname{aux}_{s}\right) \mid s \in\left[L_{\mathrm{B}}\right]\right\},
$$

where aux contains the information as in conventional CRL and $L_{\mathrm{B}}$ is the number of serial numbers recorded in $\mathrm{SCRL}^{\mathrm{B}}$. A CRL at level $k, 1 \leq k<\mathrm{B}$, is called a slave CRL and has the following form

$$
\mathrm{sCRL}^{k}:=\left\{\left(S N_{s, 1}^{k+1}, S N_{s, \text { max }}^{k+1}, H\left(\mathrm{sCRL}_{s}^{k+1}\right)\right) \mid s \in\left[L_{k}\right]\right\},
$$

where $S N_{s, 1}^{k+1}$ and $S N_{s, \text { max }}^{k+1}$ are the minimal serial number and the maximal serial number contained in $S C R L_{s}^{k+1}$ respectively and $L_{k}$ is the number of sCRL's associated with $S C R L^{k}$. We call the top-level list the master CRL. A master CRL has the 


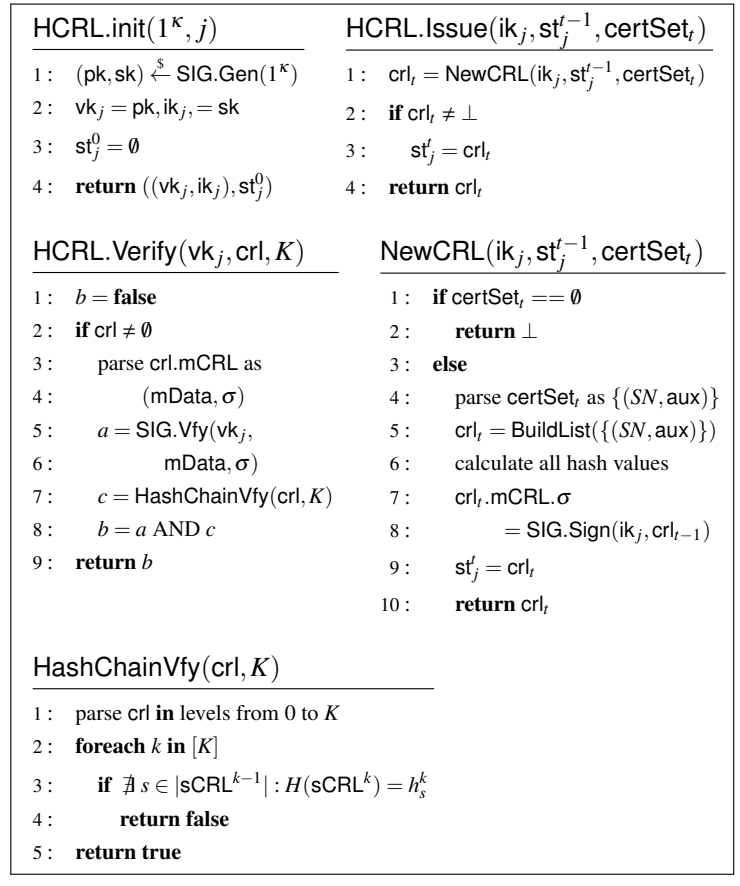

Figure 5: Algorithms in HCRL

following form

$$
\mathrm{mCRL}:=\left(\mathrm{sCRL}^{0}, \text { issuerlnfo, } \sigma\right),
$$

where $\sigma=\operatorname{SIG}$.Sign $\left(\mathrm{ik}, \mathrm{sCRL}^{0} \|\right.$ issuerlnfo), and SIG = (SIG.Gen, SIG.Sign, SIG.Vfy) the digital signature scheme chosen by the CA. In the following discussion, we use $\mathrm{mData}$ to denote $\left(\mathrm{sCRL}^{0} \mid\right.$ issuerlnfo $)$ in $\mathrm{mCRL}$ so $\mathrm{mCRL}=$ (mData, $\sigma)$.

Following the syntax of CRL in Def. 5, the major algorithms HCRL.init(), HCRL.Issue(), HCRL.Verify() and NewCRL() are summarized in Figure 5. The algorithm $\operatorname{BuildList}(\{(S N$, aux $)\})$ is almost identical to a typical balanced tree construction algorithm with $S N$ as the index, so we omit it due to space.

If the user can download the content relevant to the $S N$ that he wants to check along a path from the master CRL instead of pulling down the whole CRL, a tremendous reduction of the RAM/ROM consumption and download time can be observed. More specifically, the sub-algorithm HashChainVfy() actually allows the IoT devices to store only one SCRL at a time during the verification. Furthermore, due to the well-ordered $S N$ in each level and the bottom blocks, the time to search for a given $S N$ is also largely shortened. In the simplest case of HCRL, we can formalize the following parameter configuration theorem to strike the balance between bandwidth and RAM consumption. Let $C$ be the total number of certificates, $\rho$ the probability that one certificate becomes revoked before expiration, $M$ the byte-size of one item in $\mathrm{MCRL}$ and $S$ the byte-size of one item in $\mathrm{SCRL}^{\mathrm{B}}$.

THEOREM 1. If $L=2$, given $C, \rho, M$ and $S$, to minimize download size, the optimal number of items in each block at level $\mathrm{B}$ is

$$
x=\sqrt{\frac{C \cdot \rho \cdot M}{S}}
$$

PROOF. If $L=2$, the CRL user only has to download the master CRL and one slave CRL block, so the size of downloaded content in total is

$$
f(x)=\frac{C \cdot \rho}{x} \cdot M+x \cdot S
$$

By setting $\frac{\partial f(x)}{\partial x}=0$ and solving for $x$, we get (1).

Since adjusting these parameters in more complicated cases corresponds to a multi-variable optimization problem, and its exact analytic solution can be too complex to be adopted in real world, we develop the following empirical relation between $C, \rho, M, S$ and $x$.

THEOREM 2. Let $\xi$ be the byte-size of issuerlnfo $\| \sigma$ in mCRL. To minimize the download size, the optimal number of items in each block at level B can be approximated by solving for $x$ in the following equation.

$$
x^{\mathrm{B}+1}-\frac{\xi}{S} \cdot x^{\mathrm{B}}-C \cdot \rho \cdot\left(\frac{M}{S}\right)^{\mathrm{B}}=0
$$

The experimental results for HCRL with number of levels = 2, 3 and $4(B=1,2,3$ respectively) illustrating the efficiency of the protocol and the quality of approximation are presented in Section 5. The number of items in SCRL at level 1 to (B-1) can be computed as $\frac{S}{M} \cdot x$.

Finally, we summarize the security of HCRL in the following theorem.

THEOREM 3. If the hash function $H$ is collision-resistant and the signature scheme SIG is strongly existential unforgeable against chosen message attacks, then $\mathrm{HCRL}$ is a secure CRL protocol with integrity in the sense of Definition 6.

Proof. (Sketch) If the adversary $\mathscr{A}$ output a CRL crl ${ }^{*} \notin \mathscr{Q}$ that passes the verification, which breaks the integrity, it means that at least one of the following events happened.

- It produced a new signature on a CRL. This means a signature forgery.

- It uses an old signature but the signature is valid on a different CRL. This means a hash collision has been found, with the old CRL data as $m_{0}$ and the different CRL data as $m_{1}$.

By summarizing these two cases, Theorem 3 is proved.

\section{Bloom Filter based CRL with white list}

To check whether a certificate has been revoked in a CRL is equivalent to test whether its serial number belongs to the set of serial numbers of revoked certificates. If membership test can be made more efficient with respect to RAM and use of bandwidth, so can the CRL protocol. The main idea here is to pack the set into a constant sized data structure, so that even when the total number of revoked certificates increases, the time for downloading CRL and checking remains unchanged. Furthermore, this data structure should be much smaller than a conventional CRL for the identical serial number set. 


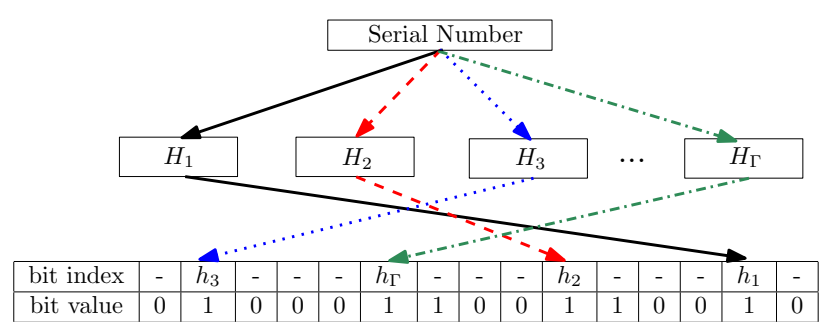

Figure 6: Bloom filter from serial numbers

\begin{tabular}{|c|c|}
\hline BFCRL.init $\left(1^{\kappa}, j\right.$, BFLen $)$ & BFCRL.Issue $\left(\mathrm{ik}_{j}, \mathrm{st}_{j}^{t-1}\right.$, certSet $_{t}$, allCerts $)$ \\
\hline 1: $(\mathrm{pk}, \mathrm{sk}) \stackrel{\mathrm{s}}{\leftarrow} \mathrm{SIG} \cdot \operatorname{Gen}\left(1^{\kappa}\right)$ & $1: \quad$ if $\mathrm{st}_{j}^{t-1}=\emptyset$ \\
\hline $2: \mathrm{vk}_{j}=\mathrm{pk}, \mathrm{ik}_{j},=\mathrm{sk}$ & 2: $\quad$ return $\perp$ \\
\hline $3: \quad w=\emptyset$ & 3: else \\
\hline 4: $B F=\{0\}^{\text {BFLen }}$ & 4: foreach $S N$ in certSet $t_{t}$ \\
\hline 5: $\quad \mathbf{s t}_{j}^{0}=(B F, w)$ & foreach $H_{\gamma}$ in $\mathbb{H}_{\mathrm{BF}}$ \\
\hline $6: \quad \operatorname{return}\left(\left(\mathrm{vk}_{j}, \mathrm{ik}_{j}\right), \mathrm{st}_{j}^{0}\right)$ & set the bit to 1 at $H_{\gamma}(S N)$ in st $_{j}^{t-1} \cdot B F$ \\
\hline & foreach $S N$ in allCerts $\backslash$ certSet $_{t}$ \\
\hline BFCRL.Verify $\left(\mathrm{vk}_{j}, \mathrm{crl}\right)$ & 8: $\quad$ if $S N$ is positive in $\mathrm{st}_{j}^{t-1} \cdot B F$ \\
\hline 1: parse crl as st, $\sigma$ & $9: \quad \mathrm{st}_{j}^{t-1} \cdot w=\mathrm{st}_{j}^{t-1} \cdot w \cup\{S N\}$ \\
\hline 2: $\quad$ return SIG.Sign $\left(\mathrm{vk}_{j}, \mathrm{BF}, \sigma\right)$ & $10: \mathbf{s t}_{j}^{t}=\mathbf{s t}_{j}^{t-1}$ \\
\hline & 11: $\quad \sigma=\operatorname{SIG} . \operatorname{Sign}\left(\mathrm{ik}_{j}, \mathrm{st}_{j}^{t}\right)$ \\
\hline & 12: $\quad \mathrm{crl}=\left(\mathrm{st}_{j}^{t}, \sigma\right)$ \\
\hline & 13: return crl \\
\hline
\end{tabular}

Figure 7: Algorithms in BfCRL

Bloom filter was first introduced by Bloom [3] for efficient membership testing. A Bloom filter $B F_{\mathbb{D}}$ for a given data set $\mathbb{D}$ is a bit array with each of its bit being set to 0 by default. Let $\mathbb{H}_{\mathrm{BF}}=\left\{H_{\gamma}\right\}$ be a set of hash function bound with $\mathrm{BF}_{\mathbb{D}}$. Each element $d \in \mathbb{D}$ is hashed with every $H_{\gamma} \in \mathbb{H}_{\mathrm{BF}}$ to a bit-location $H_{\gamma}(d)$ in $\mathrm{BF}_{\mathbb{D}}$. The bit at this location is set to 1 thereby. To test whether a given element $d^{*}$ is in $\mathbb{D}$, we can simply check whether every bit-location $H_{\gamma}\left(d^{*}\right)$ is 1 in $\mathrm{BF}_{\mathbb{D}}$. If so, according to $B F_{\mathbb{D}}, d^{\prime}$ is in $\mathbb{D}$. However, this test result has one-sided error rate. While always being correct for $d \in \mathbb{D}, \mathrm{BF}_{\mathbb{D}}$ can output TURE for a $b^{*} \notin \mathbb{D}$. This error is called false positive and its probability $\operatorname{Pr}\left(\mathrm{BF}_{\mathbb{D}}, \mathbb{D}, \mathbb{H}_{\mathrm{BF}}\right)$ depends on the bit-length of $\mathrm{BF}_{\mathbb{D}}$, the number of elements in $\mathbb{D}$ and number of distinct hash functions in $\mathbb{H}_{\mathrm{BF}}$. The relation can be expressed by the equation 3 below [10]. Here $|X|$ means bit-length of $X$ if $X$ is a bit array or number of elements in $X$ if $X$ is a set.

$$
\operatorname{Pr}\left(\mathrm{BF}_{\mathbb{D}}, \mathbb{D}, \mathbb{H}_{\mathrm{BF}}\right)=\left(1-\left(1-\frac{1}{\left|\mathrm{BF}_{\mathbb{D}}\right|}\right)^{\left|\mathbb{H}_{\mathrm{BF}}\right| \cdot|\mathbb{D}|}\right)^{\left|\mathbb{H}_{\mathrm{BF}}\right|}
$$

Figure 6 illustrated how a serial number is recorded in a Bloom filter.

Similar to HCRL, the challenge in parameter choices is also to strike the balance between RAM efficiency and security, while constraining false positive rate. Fortunately, once a part of the parameters are fixed, we can directly use Equation (3) to derive other parameters. For a Bloom filter based CRL without white list, an optimal solution can be obtained by using methods proposed in [18]. As a concrete example, with the false positive probability equal to $10^{-9}$ (i.e., one mistake made per 1 billion non-revoked certificates on average) and 30

\begin{tabular}{|c|c|c|r|r|}
\hline \# revoked cert. & false pos. & \# hash func. & BF CRL size & CRL size \\
\hline $10^{3}$ & $10^{-9}$ & 30 & $5.74 \mathrm{~KB}$ & $36.54 \mathrm{~KB}$ \\
\hline $10^{4}$ & $10^{-9}$ & 30 & $53.13 \mathrm{~KB}$ & $361.74 \mathrm{~KB}$ \\
\hline $10^{5}$ & $10^{-9}$ & 30 & $522.10 \mathrm{~KB}$ & $3613.69 \mathrm{~KB}$ \\
\hline $10^{6}$ & $10^{-9}$ & 30 & $5.14 \mathrm{MB}$ & $35.28 \mathrm{MB}$ \\
\hline $10^{7}$ & $10^{-9}$ & 30 & $51.42 \mathrm{MB}$ & $350.12 \mathrm{MB}$ \\
\hline
\end{tabular}

Table 2: Asymtotic performance data of Bloom filter based CRL without white list.

\begin{tabular}{|c|c|r|r|}
\hline \#cert. & \# revoked cert. & BF size & w.list size \\
\hline $10^{4}$ & $10^{3}$ & $2.79 \mathrm{~KB}$ & $0.18 \mathrm{~KB}$ \\
\hline $10^{5}$ & $10^{4}$ & $23.63 \mathrm{~KB}$ & $1.8 \mathrm{~KB}$ \\
\hline $10^{6}$ & $10^{5}$ & $232.03 \mathrm{~KB}$ & $18 \mathrm{~KB}$ \\
\hline $10^{7}$ & $10^{6}$ & $2.26 \mathrm{MB}$ & $0.18 \mathrm{MB}$ \\
\hline $10^{8}$ & $10^{7}$ & $22.61 \mathrm{MB}$ & $1.8 \mathrm{MB}$ \\
\hline
\end{tabular}

Table 3: Asymtotic performance data of BfCRL with a white list of valid but false positive certificates.

distinct hash functions, other parameters will be as in Table 2 . By CRL size we mean the size of an conventional CRL for the same number of revoked certificates. Note that such a Bloom filter CRL requires only $\frac{1}{7}$ RAM usage of a conventional CRL.

We now present BfCRL, our Bloom filter based CRL protocol. Our idea to eliminate false positivity is straight forward: we introduce into the CRL a sorted white list of serial numbers, which belong to those valid certificates issued by the same CA but are found false positive in the Bloom filter. More precisely, CA will first build a Bloom filter BF from the revoked certificates and then go through all of the valid certificates remaining. If any serial number $S N$ of the valid certificates is found positive in BF, CA will add it to a white list $w$. Finally, CA will sign on $\mathrm{BF}$ and $w$. The algorithms in BfCRL to initialize, issue and verify a CRL can be found in Figure 7, where allCerts is the set of the serial number of all the certificates issued by the CA, allCerts $\backslash$ certSet $_{t}$ means the set of valid certificates remaining and $w$ is the white list. A signature scheme is still needed for integrity protection.

Compared with data in Table 2, it can be observed in Table 3 that more space can be saved by using white lists. Here we assume that $10 \%$ of the certificates have been revoked. If we have a Bloom filter with 30 hash functions, an original false positive rate $\varepsilon_{P}(\kappa)$ being $10^{-3}$ but we add a white list to completely eliminate the false positivity, the resulting BfCRL in Table 3 is about $50 \%$ smaller than the naive Bloom filter based CRL in Table 2. Moreover, the certificate status validation with BfCRL is now without any false positive case. If a $S N$ is found in the white list, it belongs to a valid certificate.

The following theorem illustrate the security of our BfCRL. The proof of Theorem 4 is similar to that of Theorem 3, so we omit it due to space.

THEOREM 4. If all of the hash functions in $\mathbb{H}_{\mathrm{BF}}$ is collision-resistant, the false positive probability is bound by $\varepsilon_{P}(\kappa)$ and the signature scheme SIG is strongly existentialunforgeable against chosen message attacks, then BfCRL is a secure CRL protocol with integrity in the sense of Definition 6. 


\begin{tabular}{|l|l|l|l|l|l|}
\hline \multicolumn{2}{|c|}{ \#revoked certificates } & $10^{3}$ & $10^{4}$ & $10^{5}$ & $10^{6}$ \\
\hline \hline \multirow{2}{*}{ 2-HCRL } & Level 0 & 1,560 & 4,280 & 13,052 & 40,660 \\
& Level 1 & 1,537 & 4,297 & 13,033 & 40,657 \\
\hline \multirow{3}{*}{ 3-HCRL } & Level 0 & 744 & 1,220 & 2,444 & 4,960 \\
& Level 1 & 681 & 1,225 & 2,381 & 4,965 \\
& Level 2 & 721 & 1,225 & 2,425 & 4,993 \\
\hline \multirow{3}{*}{ 4-HCRL } & Level 0 & 529 & 744 & 1,084 & 1,832 \\
& Level 1 & 409 & 681 & 1,089 & 1,769 \\
& Level 2 & 545 & 681 & 1,089 & 1,769 \\
& Level 3 & 529 & 721 & 1,081 & 1,801 \\
\hline
\end{tabular}

Table 4: Single block size (in bytes) of HCRL of different level settings.

\section{PERFORMANCE EVALUATION}

\section{Test Bench and Metrics}

We implemented HCRL and conventional CRL in JAVA with Bouncy Castle Cryptography library ${ }^{3}$. The experiments were run on a Raspberry Pi 2 Model B with LAN access. The signature scheme we chose is RSA-2048 with SHA-256 and the hash function used for the HCRL data is SHA-256. Each $S N$ has a length of 18 bytes. The auxiliary information associated with each $S N$ in the slave CRL at the bottom level contains only the date of revocation (unix epoch time in 6 bytes), so the the total length of each item at SCRL is 24 bytes. And the length of each item in all non-bottom nodes is 68 bytes.

The metrics we used are 1) the size of CRL data to be downloaded, 2) RAM consumption, 3) size of data to be hashed and 4) search complexity. We separate 1) and 2) because the IoT end-device can re-use part of its RAM by keeping only one CRL block with book keeping information in its RAM and discarding used ones during the verification. The data to be hashed and the complexity of the search process determine the latency caused by the computation at IoT end-devices.

\section{Result Summary}

We use 2-HCRL, 3-HCRL and 4-HCRL to denote HCRL with 2 levels, 3 levels and 4 levels respectively. As can be observed in Figure 8, all HCRL outperform conventional CRL significantly at all metrics.

As the number of level increases, given a fixed number of revoked certificates, the block size of CRL decreases, so do the RAM consumption. Here we exclude the RAM needed for communication and cryptographic operations. The exact size (in bytes) of each block at each level for different number of revoked certificates are shown in Table 4, the maximal of which is only $40660 \mathrm{~B}(\approx 40 \mathrm{~KB})$ per block in 2 -CRL for 1 million revoked certificates, i.e., a single block of 2-HCRL, 3-HCRL or 4-HCRL can well fit into the RAM of class 2 IoT devices in Tabel 1.

Moreover, the time to search for the given $S N$ in HCRL is greatly reduced, since the items are ordered by $S N$ in each block and the minimal and maximal are used to enable early interruption in search. This also confirms our analysis of search complexity, i.e., $\mathscr{O}(\log (C \cdot \rho))$ for HCRL vs. $\mathscr{O}(C \cdot \rho)$ for conventional CRL.

\footnotetext{
${ }^{3}$ https://www.bouncycastle.org/
}
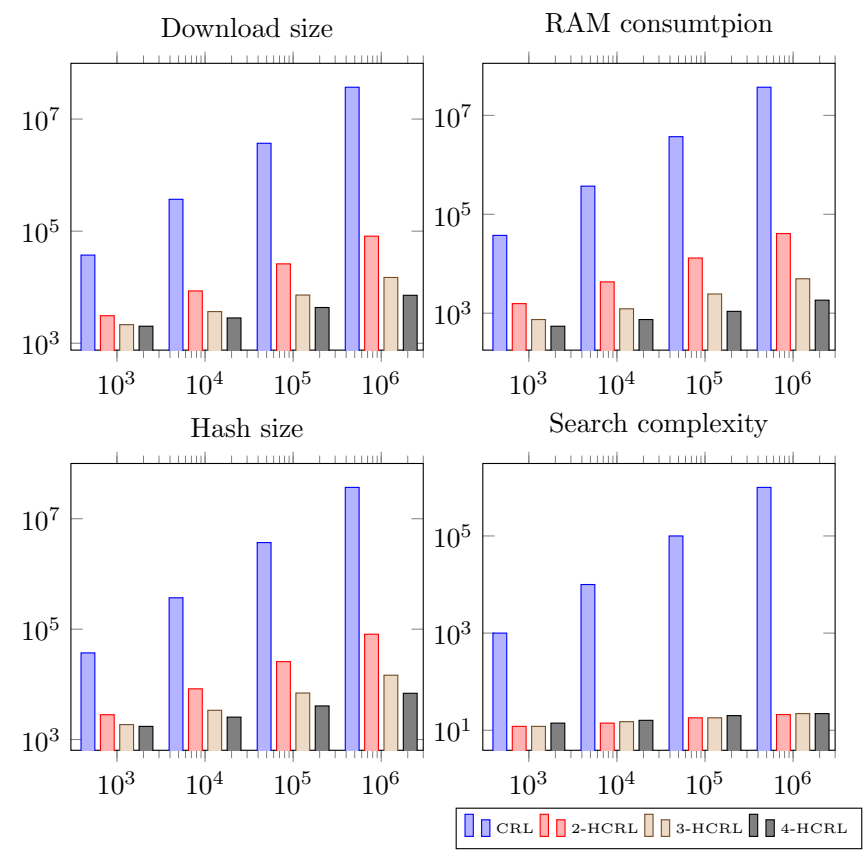

Figure 8: Performance of $C R L, 2-, 3$ - and 4-HCRL.

The $\mathrm{X}$-coordinate represents the number of revoked certificates. The y-coordinate of 'Search complexity' is the number of comparisons, and that of the other three diagrams is the number of bytes with logarithmic scale.

\section{RELATED WORKS}

Authenticated key exchange protocols and PKI. Being proposed by Diffie and Hellman [8] in their celebrated work, public cryptography based key exchange protocols over insecure channels are now an indispensable technology to protect valuable data. In various theoretical security models for authenticated key exchange (AKE) or channel establish protocols [2] [12], an authentication infrastructure is assumed to exist for the authentication of entities. In the real world, whenever DTLS or TLS is introduced for secure key exchange and data confidentiality, Public Key Infrastructure (PKI) [6] is the standardized foundation for authenticity of entities, unless the communicating parties possess pre-shared cryptographic keys [9].

Other authentication infrastructures. New authentication infrastructures, such as Keyless Signatures' Infrastructure [5] or block-chain based authentication [17] might be prominent in the future. However, completely abandoning the existing infrastructure or high investment on improving the IoT enddevice's power might not be the price that enterprises are willing to pay.

Approaches for performance improvement of PKI. It can also improve the performance of PKI in general to replace heavyweight cryptographic primitives with lightweight ones. LPKI [19] is a typical example of this approach, where RSA signature is replaced with elliptic curve based signature [7] and TLS handshake with HMQV. Note that the approach taken by LPKI [19] is orthogonal to ours since our CRL protocols do 
not rely on any specific digital signature scheme or encryption scheme. On the other hand, both approaches can be combined for even more performance gain. Previous research showed the quantitative methods for optimizing bloom filter parameters when compressing CRL information [18]. Such Bloom filter based CRLs were also suggested for the browser [14], V2X communication [18] and advanced metering networks [15]. All of them constrained but did not eliminate false positivity.

\section{CONCLUSION AND FUTURE WORK}

In this paper, we constructed highly efficient and provably secure CRL protocols, which can be applied to IoT devices of various computation power. Our evaluation show that HCRL and BfCRL achieve significantly low RAM and bandwidth consumption compared to conventional CRL [6]. We leave it in future research to design other lightweight components for authentication infrastructure such as PKI, so that IoT enddevices can enjoy security guarantee comparable with that of conventional computers.

\section{REFERENCES}

1. Ala Al-Fuqaha, Mohsen Guizani, Mehdi Mohammadi, Mohammed Aledhari, and Moussa Ayyash. 2015. Internet of things: A survey on enabling technologies, protocols, and applications. IEEE Communications Surveys \& Tutorials 17, 4 (2015), 2347-2376.

2. Mihir Bellare and Phillip Rogaway. 1994. Entity Authentication and Key Distribution. In Advances in Cryptology - CRYPTO'93 (Lecture Notes in Computer Science), Douglas R. Stinson (Ed.), Vol. 773. Springer, 232-249.

3. Burton H Bloom. 1970. Space/time trade-offs in hash coding with allowable errors. Commun. ACM 13, 7 (1970), 422-426.

4. C. Bormann, M. Ersue, and A. Keranen. 2014. Terminology for Constrained-Node Networks. RFC 7228 (Informational). (March 2014). http://www.ietf.org/rfc/rfc7228.txt

5. Ahto Buldas, Andres Kroonmaa, and Risto Laanoja. 2013. Keyless Signatures' Infrastructure: how to build global distributed hash-trees. In Nordic Conference on Secure IT Systems. Springer, 313-320.

6. D. Cooper, S. Santesson, S. Farrell, S. Boeyen, R. Housley, and W. Polk. 2008. Internet X.509 Public Key Infrastructure Certificate and Certificate Revocation List (CRL) Profile. RFC 5280 (Proposed Standard). (May 2008). http://www.ietf.org/rfc/rfc5280.txt

7. A. Deacon and R. Hurst. 2007. The Lightweight Online Certificate Status Protocol (OCSP) Profile for High-Volume Environments. RFC 5019 (Proposed Standard). (Sept. 2007). http://www.ietf.org/rfc/rfc5019.txt

8. Whitfield Diffie and Martin Hellman. 1976. New directions in cryptography. IEEE transactions on Information Theory 22, 6 (1976), 644-654.
9. P. Eronen and H. Tschofenig. 2005. Pre-Shared Key Ciphersuites for Transport Layer Security (TLS). RFC 4279 (Proposed Standard). (Dec. 2005).

http://www.ietf.org/rfc/rfc4279.txt

10. Li Fan, Pei Cao, Jussara Almeida, and Andrei Z Broder. 2000. Summary cache: a scalable wide-area web cache sharing protocol. IEEE/ACM transactions on networking 8, 3 (2000), 281-293.

11. Internet Engineering Task Force. 2008. Transport Layer Security Version 1.2. RFC 5246. (2008). https://tools.ietf.org/html/rfc5246.

12. Tibor Jager, Florian Kohlar, Sven Schäge, and Jörg Schwenk. 2012. On the security of TLS-DHE in the standard model. In Advances in Cryptology-CRYPTO 2012. Springer, 273-293.

13. Aron Laszka, Abhishek Dubey, Michael Walker, and Douglas C. Schmidt. 2017. Providing privacy, safety, and security in IoT-based transactive energy systems using distributed ledgers. In Proceedings of the Seventh International Conference on the Internet of Things, IOT 2017, Linz, Austria, October 22-25, 2017. 13:1-13:8.

14. Yabing Liu, Will Tome, Liang Zhang, David Choffnes, Dave Levin, Bruce Maggs, Alan Mislove, Aaron Schulman, and Christo Wilson. 2015. An end-to-end measurement of certificate revocation in the web's PKI. In Proceedings of the 2015 Internet Measurement Conference. ACM, 183-196.

15. Mohamed Mahmoud, Kemal Akkaya, Khaled Rabieh, and Samet Tonyali. 2014. An efficient certificate revocation scheme for large-scale AMI networks. In Performance Computing and Communications Conference (IPCCC), 2014 IEEE International. IEEE, $1-8$.

16. Ralph C Merkle. 1987. A digital signature based on a conventional encryption function. In Conference on the Theory and Application of Cryptographic Techniques. Springer, 369-378.

17. Axel Moinet, Benoît Darties, and Jean-Luc Baril. 2017. Blockchain based trust $\&$ authentication for decentralized sensor networks. arXiv preprint arXiv: 1706.01730 (2017).

18. Giovanni Rigazzi, Andrea Tassi, Robert J Piechocki, Theo Tryfonas, and Andrew Nix. 2017. Optimized Certificate Revocation List Distribution for Secure V2X Communications. arXiv preprint arXiv: 1705.06903 (2017).

19. Mohsen Toorani and A Beheshti. 2008. LPKI-a lightweight public key infrastructure for the mobile environments. In Communication Systems, 2008. ICCS 2008. 11th IEEE Singapore International Conference on. IEEE, 162-166.

20. Guest Writer. 2017. The 5 Worst Examples of IoT Hacking and Vulnerabilities in Recorded History. https://www.iotforall.com/ 5-worst-iot-hacking-vulnerabilities/. (May 2017). 